\title{
COMBAT SIMULATION FRAMEWORK INCLUDING CONTINUOUS DETECTION SYSTEM
}

\author{
Ham, W. K.; Kwon, Y. \& Park, S. C.* \\ Department of Industrial Engineering, Ajou University, \\ San 5, Woncheon-dong, Yeongtong-gu, Suwon, Republic of Korea \\ E-Mail: lunacy@ajou.ac.kr, yk73@ajou.ac.kr, scpark@ajou.ac.kr ("Corresponding author)
}

\begin{abstract}
This paper presents a framework for an anti-air engagement simulation that includes continuous detection systems. According to resolutions, military simulations can be categorized into four different levels; campaign (war-game), mission, engagement, and engineering (natural-phenomenon). While the campaign and mission level systems are represented by discrete-event systems and the engineering level systems are represented by continuous-state systems, the engagement level systems may include both characteristics of the discrete and continuous systems. An engagement level system includes multiple combat entities represented by discrete-event systems; however, various performance parameters of combat entities (i.e. detection probability) belong to continuous-state systems. Although the detection probability can be computed by equations considering the synthetic environment, it cannot be directly used in a discrete-event system. To cope with the problem, we propose a glimpse probability suitable for the discrete-event systems and a construction procedure. The proposed framework is designed to be compatible with high-level architecture technology, and applied to antiair engagement simulations.

(Received in June 2013, accepted in March 2014. This paper was with the authors 1 month for 1 revision.)
\end{abstract}

Key Words: Continuous-State System, Discrete-Event System, Engagement Simulation, Glimpse Probability, High-Level Architecture, Synthetic Environment

\section{INTRODUCTION}

Owing to the changes that are creating future paradigms of combat, along with the remarkable progress in the development of weapon systems, military forces are facing unprecedented, varied, and complex requirements in areas such as new weapon development and tactical deployment. The military resources that are available to address these needs, though, are limited as compared to the requirements. DM\&S (Defence modelling and simulation) has emerged as a key to resolving this disparity between resources and requirements. DM\&S facilitates efficient planning for resource consumption by verifying whether a given plan meets the requirements before the resources are committed [1]. Thus, DM\&S is an inevitable trend in the design and operation of military technology [2]. Recently, many countries have sought to apply DM\&S beyond such traditional applications as training and analysis, e.g., applying it to the cycle of weapon systems acquisition [3].

$\mathrm{DM} \& \mathrm{~S}$ is classified according to the level of detail of the representation, which is defined by the DoD (Department of Defence) in four levels: campaign, mission, engagement, and engineering (see Table I). An engagement simulation model, which is the level addressed in this paper, describes the sophisticated behaviours and functions of weapon systems for short durations ranging from minutes to hours, but it is not concerned with tactical commands relatively. The expected outcomes of an engagement simulation model include issues such as survivability, vulnerability, and detection-ability of weapon systems [4]. According to the characteristics of an engagement simulation model, the model has to represent the design properties and behaviours of each weapon system that is involved in the combat. However, it is possible that the operational results of weapon systems may not reflect the systems' own 
design factors in real engagements, because of environmental effects. If we look at the history of warfare, we see that environmental effects can be a decisive factor in determining the success or failure of operations, and this is still the case, even though the technologies of today's weapon systems have become highly developed [5]. For this reason, in an engagement simulation model, the synthetic environment must reflect the environmental effects upon the simulation entities, and the simulation results must be able to be changed to reflect the effects of the synthetic environment [6].

Table I: Defence modelling and simulation models.

\begin{tabular}{|c|c|c|c|c|}
\hline Level & Scope & Main Description & Output & System Type \\
\hline Campaign & Nation & $\begin{array}{c}\text { - Threat } \\
\text { - Mission Type }\end{array}$ & $\begin{array}{c}\text { - War Result } \\
\text { - Military Loss }\end{array}$ & Discrete \\
\hline Mission & Division & $\begin{array}{c}\text { - Threat, } \\
\text { - Mission Type }\end{array}$ & $\begin{array}{c}\text { - Loss Rate } \\
\text { - Mission Efficiency }\end{array}$ & Discrete \\
\hline Engagement & Small Unit & $\begin{array}{c}\text { - Operation } \\
\text { - System Capability }\end{array}$ & $\begin{array}{c}\text { - Survivability } \\
\text { - Vulnerability }\end{array}$ & $\begin{array}{c}\text { Discrete }+ \\
\text { Continuous }\end{array}$ \\
\hline Engineering & $\begin{array}{c}\text { Weapon } \\
\text { System }\end{array}$ & - System Capability & $\begin{array}{c}\text { - Capacity } \\
\text { - Detection probabilities }\end{array}$ & Continuous \\
\hline
\end{tabular}

In the engagement simulation model, the adoption of synthetic environment based systems causes more difficulties in representing combats than other models. The campaign and mission models describe behaviours of combat entities for the representation of commands and tactics by the discrete-event system. The engineering model, such as a detection system that considers environmental effects, describes system performance by the continuous-state system. But the engagement simulation model requires both systems to be overlapped as applying continuous-state systems to a discrete-event system base, since behaviours of combat entities are represented by the discrete-event system and detection states among combat entities are represented by the continuous-state system. The problem is that though the detection probability belonging to a continuous system can be computed by the performance equation considering the synthetic environment, it cannot be directly used in the discreteevent system performing detection activities at every discrete-event repetitively. For example, if a detection probability between an entity ' $\mathrm{A}$ ' and an entity ' $\mathrm{B}$ ' is continuously applied, and then the result always will be that ' $\mathrm{A}$ ' detects ' $\mathrm{B}$ '. Thus, operations of the continuous detection systems have to be reprocessed for applying to the discrete-event simulation system.

Previous research and existing tools for simulation system frameworks have been reported for various fields, such as engineering design [7], manufacturing simulation [8-10], and traffic control simulation. But these frameworks are for simulations which require only discreteevents, rather than including continuous-state systems. For DM\&S, a number of simulation tools are currently available, such as VBS2 (Virtual battle space 2), AnyLogic, and ARENA. These existing tools, though, are not adequate for an engagement simulation that includes continuous-state systems, because they do not allow users to access their operation system. In other words, the execution of engagement simulations using existing tools is limited within the tool-provided functional scope of the discrete-event base [11]. Meanwhile, the simulation systems are developed as a new system, in general, to beyond the limitation of existing tools and to meet users' requirements. There is research for the dedicated framework for DM\&S to guide the construction of a simulation system for efficient development. Park et al. researched a framework for a small-scale engagement simulation [12], which focuses on a methodology for the description of the behaviours of simulation entities, using DEVS (Discrete-event system specification). Yildirim et al. proposed a military deployment simulation based on the discrete-event system [13], with regard to a simulation whose aim is to find the most efficient solution for the transportation of military assets. The research of Son and Kim addressed a 
simulation of torpedo evasion of an underwater vehicle and focused on the decision-making logic of the underwater vehicle's evasion tactics [14]. However, former DM\&S research also focused on the representation of behaviours of simulation entities as discrete-events, so that the results are limited in the discrete-event based system development. Although the synthetic environment and environmental effects were mentioned in the research of Park et al. [12], they were bounded within a conceptual idea. Wilcox et al. remarked that the use of the synthetic environment will be a key factor in future DM\&S and that it is necessary to obtain high fidelity and a high level of detail in an engagement simulation [15]. Hence, in anticipating the future, continuous detection systems considering a synthetic environment are a necessity that must be included in the engagement simulation model. Both the existing tools and previous research, though, have not considered DM\&S that makes use of continuous systems.

Although there have been various research results on military simulation, the engagement simulation framework including both characteristics of discrete and continuous systems has rarely been brought into focus. Thus, a military simulation that includes continuous-state systems to account for environmental effects is a field in which research is needed. To cope with the problem, this paper proposes a framework for an engagement simulation system that includes continuous detection systems by a GP (Glimpse probability) which we propose is suitable for the discrete-event systems. The proposed framework is designed to be compatible with HLA (High level architecture) technology, and the construction procedure of the GP table from the performance equation of a combat entity on the HLA based system is also provided in the framework. Using the proposed framework, we expect to clarify the issues involved in the construction of an engagement simulation system by means of continuous detection systems, whose simulation results reflect environmental effects. The framework is applied to an anti-air engagement simulation system as an example.

This paper is organized as follows. Section 2 reviews related technical background to help for understanding this paper. Section 3 describes details of the proposed framework for the anti-air engagement simulation system. Section 4 includes a description of the implementation of an example system, as a demonstration of the proposed framework. Finally, Section 5 summarizes the main conclusions of this study.

\section{TECHNICAL APPROACH}

\subsection{Detection system in anti-air engagement}

In an anti-air engagement, radar (an abbreviation for radio detection and ranging) is the most efficient detection system for searching for objects in a specific area, such as aircraft, ships, and missiles. A radar detection system uses radio waves, and is composed of two parts: a transmitter and a receiver. The transmitter emits pulses of radio waves, and the emitted radio waves propagate along a straight line in the direction in which they are emitted by the transmitter, at the speed of light. The emitted radio waves bounce off of objects that are in the path of the propagation direction, and these bounced radio waves are returned to the receiver. A radar detection system analyses the differences between the emitted radio waves and the returned radio waves in order to determine both the location of the object that the radio waves bounced off of, as well as the kind of object [16]. As the radio waves travel from the transmitter toward an object and from the object back to the receiver, they are propagated at different levels of the atmosphere, including the troposphere and the stratosphere. Although the propagation of radio waves is predictable using a mathematical equation in free space, the atmosphere of an engagement in a real battlefield is not free space. The atmosphere includes various elements that differ in terms of the time, the day, regions, heights, etc., which are 
reflected in factors such as water vapour in the troposphere, and those elements cause the phenomena of reflection, refraction, diffraction, absorption, polarization, and scattering, all of which affect the propagation of radio waves [17].

As a result of the effects of these phenomena on radio waves, these waves get attenuated. A radar detection system may be confused in its determination of the returned radio waves, because those waves may be weaker than the detection threshold of the radar system, or because they may be modulated in a way that is difficult to identify [18]. Owing to the relationship between radio waves and the atmosphere, the probability that a radar detection system will detect objects in the search area is not constant, as it is in free space. It is, rather, a value that is changeable, because of environmental effects. Therefore, the detection system in an anti-air engagement simulation must be based on a synthetic environment that reflects environmental effects in the computation of the detection probability.

\subsection{HLA based system}

HLA is the standard for a distributed system according to IEEE 1516 [19], and it is developed in order to facilitate its interoperability and reusability by M\&SCO (Modelling and simulation coordination office: an affiliate of the U.S. DoD, which leads in the development of DoD $M \& S$ standardization and the empowerment of M\&S capabilities) [20]. HLA provides a standard protocol for a distributed system that facilitates the rapid and economical construction of a larger, more realistic simulation via the integration of various applications [21]. A distributed system reduces the resources required to build a new simulation system, such as time, cost, and manpower, by promoting the reusability of the unit's applications. An HLA-based distributed system is one of the indirect communication systems that employs the P-S (publish-subscribe) paradigm for distributed event-based systems, which are composed of a server and clients. In this kind of system, clients are assumed as publishers and/or subscribers, publishers publish structured events to an event service, and subscribers express interest in particular events through subscriptions [22]. An HLA-based distributed system specifies several terms that are necessary in order to understand the data interchange of HLA; these terms are described in Table II [23, 24].

Table II: Description of HLA terms.

\begin{tabular}{|c|l|}
\hline Component & \multicolumn{1}{c|}{ Description } \\
\hline $\begin{array}{c}\text { Run-Time } \\
\text { Infrastructure (RTI) }\end{array}$ & $\begin{array}{l}\text { The software that provides common interface services during an HLA federation } \\
\text { execution for synchronization and data exchange. }\end{array}$ \\
\hline Federation & $\begin{array}{l}\text { A named set of federate applications and a common Federation Object Model (FOM) } \\
\text { that are used as a whole to achieve some specific objective. }\end{array}$ \\
\hline Federate & $\begin{array}{l}\text { An application that may be or is currently coupled with other software applications under } \\
\text { FOM document data (FDD) and an RTI. An application that supports the HLA interface } \\
\text { to an RTI and that is capable of being combined with a federation execution. }\end{array}$ \\
\hline Publish & $\begin{array}{l}\text { To announce to a federation the information that a federate may provide to the } \\
\text { federation. }\end{array}$ \\
\hline Subscribe & To announce to a federation the information that a federate wants from the federation. \\
\hline
\end{tabular}

\section{FRAMEWORK}

\subsection{Synthetic environment for radar detection system}

In order to construct such a synthetic environment for a radar detection system, EPOs (environment process object), which are the required objects of nature that must be processed for the representation of the intended environmental effects, must be derived at the beginning 
of the construction process. In this paper, EPOs that are elements of the atmospheric attenuation computation are derived as example EPOs. Atmospheric attenuation can be due to three kinds of environmental effects: rain, vapour, and oxygen attenuation. In order to compute each type of attenuation, precipitation data (collected in units of $\mathrm{mm} / \mathrm{hour}$ ) are required for the rain attenuation formula as defined by the recommendation of ITU-R (International Telecommunication Union-Radio communication) P. 838 [25]. The elements of atmospheric pressure (hectopascal) and temperature (Celsius) are required for the oxygen attenuation formula, and humidity (percent) and the former two elements are required for the vapour attenuation formula. Both the oxygen and the vapour attenuation formulas are defined according to the recommendation of ITU-R P. 676 [26].

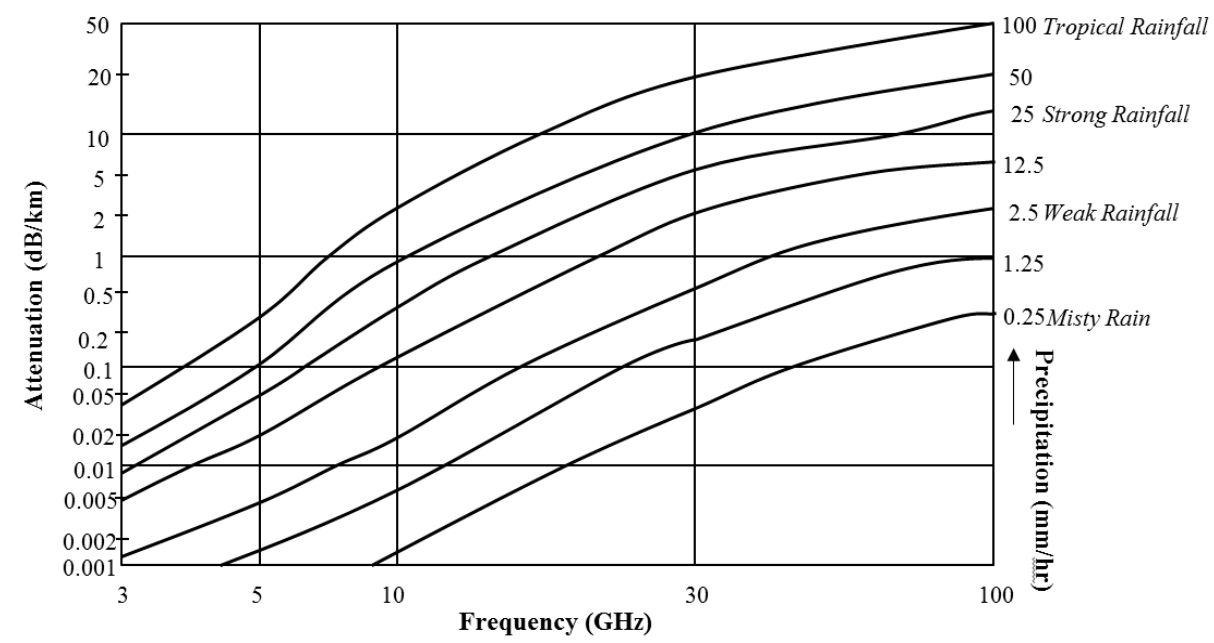

Figure 1: Rain attenuation by frequency and precipitation.

For the representation of attenuation due to environmental effects, the scientific results of rain attenuation (see Fig. 1) and those for vapour and oxygen attenuation are described (see Fig. 2).

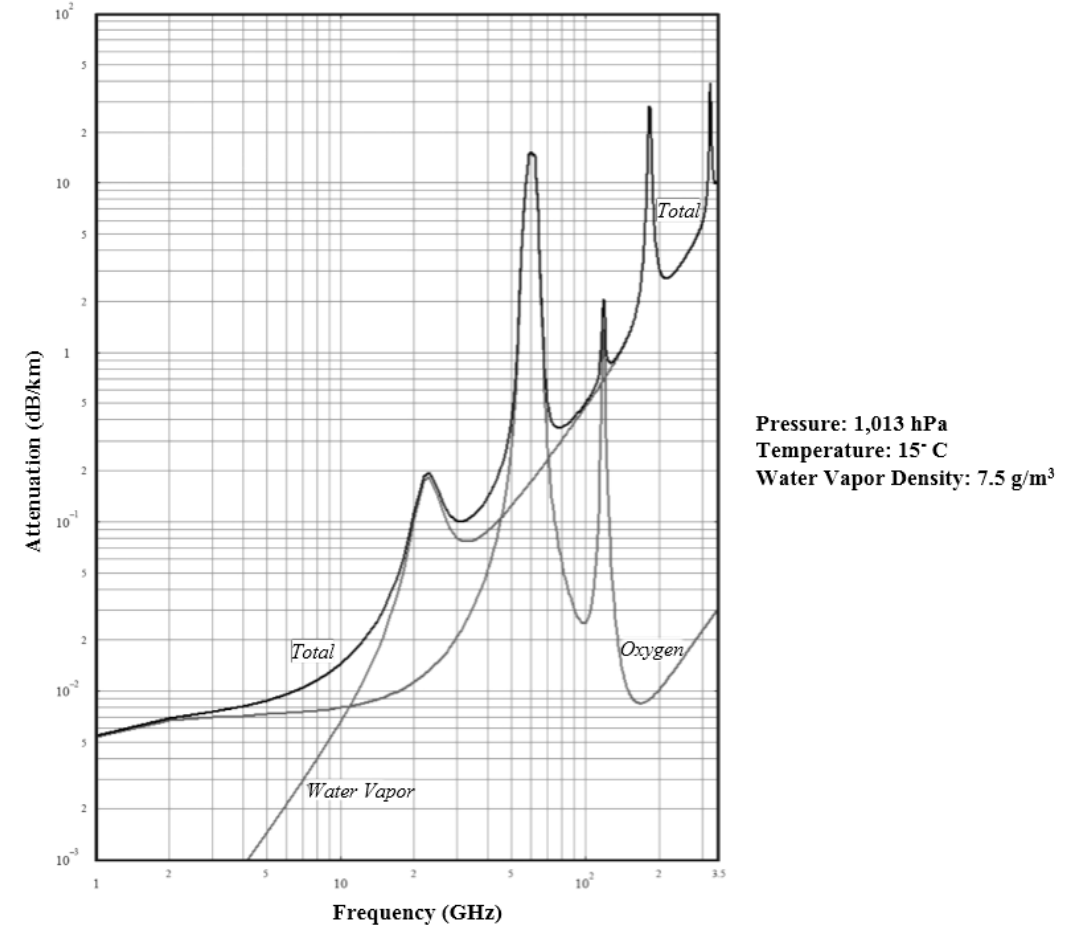

Figure 2: Vapour and oxygen attenuation by frequency. 
As shown in Fig. 1, radio waves with a frequency of $100 \mathrm{GHz}$ are attenuated as $50 \mathrm{~dB} / \mathrm{km}$ in tropical rainfall, and as $2 \mathrm{~dB} / \mathrm{km}$ in weak rainfall. The detectable area decreases by $11 \%$ and $68 \%$ by the attenuation of $2 \mathrm{~dB} / \mathrm{km}$ and $20 \mathrm{~dB} / \mathrm{km}$, respectively [27]. Based on the attenuation curves of the specific atmospheric condition shown in Fig. 2, the oxygen attenuation is about $15 \mathrm{~dB} / \mathrm{km}$ for a radio wave frequency of $50 \mathrm{GHz}$, and the vapour attenuation is about $0.2 \mathrm{~dB} / \mathrm{km}$ for a frequency of $23 \mathrm{GHz}$. The total shown in Fig. 2 is the sum of the vapour and oxygen attenuations at a given frequency, and the total attenuation of radio waves by environmental effects will thus be the sum of all the attenuation results for the three types. A synthetic environment is constructed based on the environmental data of the derived EPOs. Thus, the environmental data for the derived EPOs in this study are collected for the synthetic environment of an anti-air engagement. The collected environmental data are to be structured to suit the form of the synthetic environment, because environmental data are provided as a collection of numerical data.

\subsection{GP table}

In order to apply the detection operation of a radar system to combats of the engagement simulation, this study proposes the GP table presented in Table III, which consists of two columns: one for distance ranges, and the other for GPs. The GP indicates the detection probability in the indicated distance range, and a GP is computed using the radar performance and environmental data of the synthetic environment [28, 29]. The GP table defines the detection probabilities in terms of the distances between a radar system and objects, so it is able to be applied to the detection stages of the simulation entities in an anti-air engagement simulation. In conclusion, operation results of continuous radar systems are converted to discrete detection states using the GP table that allows applying continuous detection systems to discrete-event based combat simulations, and the distortion which is occurred by repetitive trials is prevented by using the GP table. For example, the GP table of an entity ' $A$ ' indicates a GP of an entity ' $\mathrm{B}$ ' based on the distance between $A$ and $B$, and the GP indication operation is executed once in each distance range.

Table III: Example of GP table.

\begin{tabular}{|c|c|}
\hline Distance Range (km) & GP (\%) \\
\hline $60-70$ & 20.203125 \\
\hline $50-60$ & 39.384125 \\
\hline $40-50$ & 39.859103 \\
\hline $30-40$ & 43.901131 \\
\hline $20-30$ & 40.121001 \\
\hline $10-20$ & 26.193628 \\
\hline $0-10$ & 11.617442 \\
\hline
\end{tabular}

\subsection{Engagement simulation model in distributed system}

In this study, an HLA-based distributed system is applied to the construction of the anti-air engagement simulation system, and the data interchange procedure in the system is defined. Thus, the operations allocated for the GP table's construction and utilization are clarified. The proposed simulation framework describes an HLA-based data interchange procedure for the construction of a complete GP table to be applied to an anti-air engagement simulation. In the proposed framework, the federation for an anti-air engagement simulation with a continuous radar detection system that considers the synthetic environment is designed to be composed of an RTI server and three federates: simulation, radar detection, and environment federates. The 
simulation federate has simulation entities, such as radar bases, aircraft, and missiles, and it executes the anti-air engagement simulations using the entities. The radar detection federate calculates radar detection probabilities using radar specification and environment factors, and the environment federate searches the environmental data of the synthetic environment. The operation results of each federate are exchanged via an RTI server; the data interchange procedure of the federation is explained in a sequence diagram in Fig. 3. The diagram illustrates the process of GP table construction via the cooperation among the federates; the complete GP table is applied to the engagement simulation in the simulation federate.

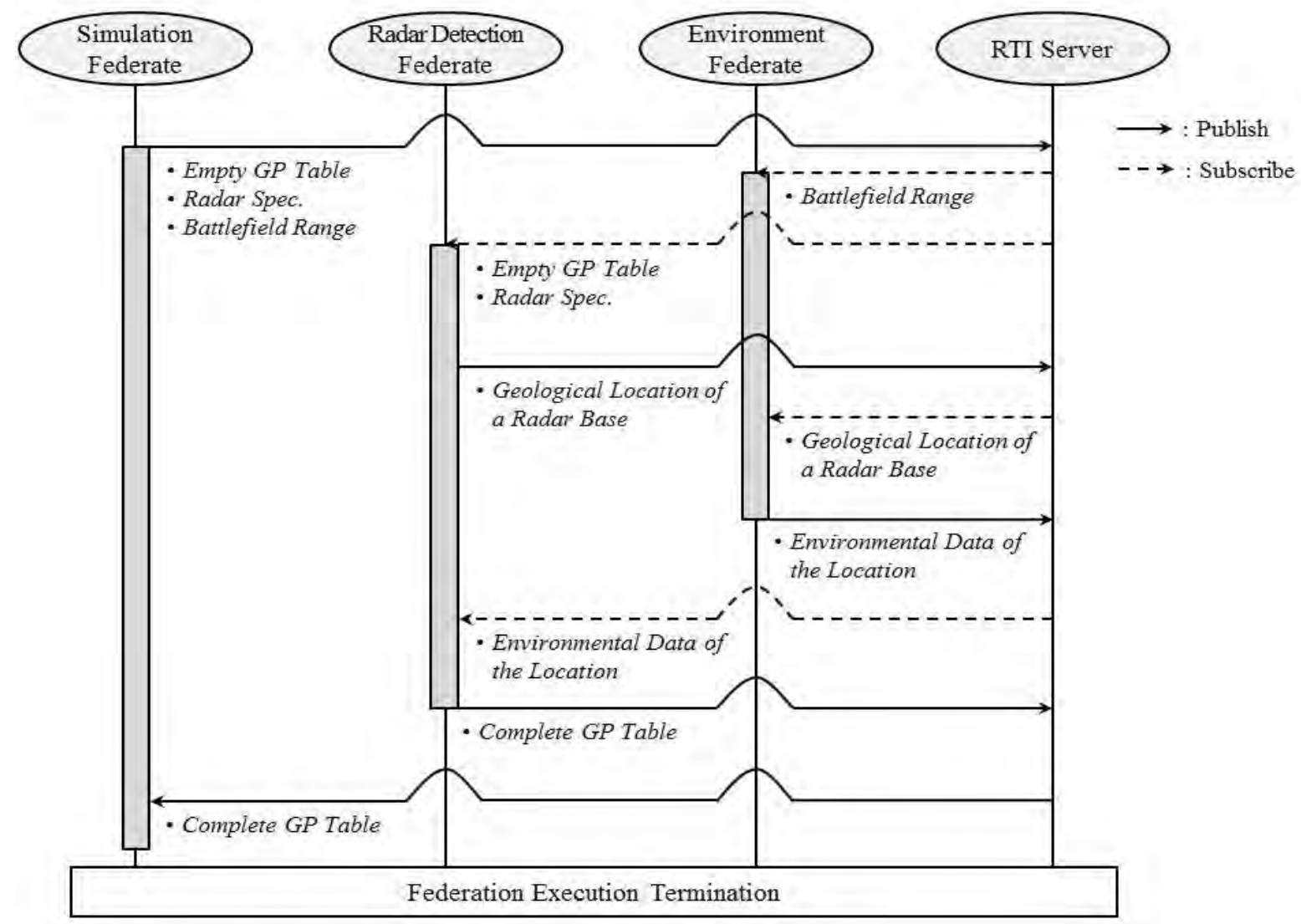

Figure 3: Sequence diagram for description of data interchange.

To offer a detailed explanation of the framework, this paper depicts the operations of each federate along with the procedure of data interchange. At the beginning of the procedure, the simulation federate publishes an empty GP table, a radar specification, and a battlefield range. The environment federate subscribes to the battlefield range and extracts the environmental data from the database for the battlefield range it subscribed to; this operation is depicted in Fig. 4. In this paper, the database of the environment federate is based on atmosphere data provided by Korea's meteorological administration. The provided data consist of the precipitation, pressure, temperature, and water vapour density of the atmosphere, and other environmental data for the Korean peninsula, which provide sufficient attributes for the calculation of the three attenuation types. The provided data are arranged according to the collection time by identifying the point in time (represented in year, month, day, and hour) at which the data were collected. The data for one collection time are arranged by the collection station so as to identify the geographical location point (represented in latitude and longitude) of the data. Thus, one data collection station contains environmental data for the atmosphere attributes at the time of collection. As shown in Fig. 4, the environment federate extracts the stations that are within the time and geographical location of the battlefield range. 


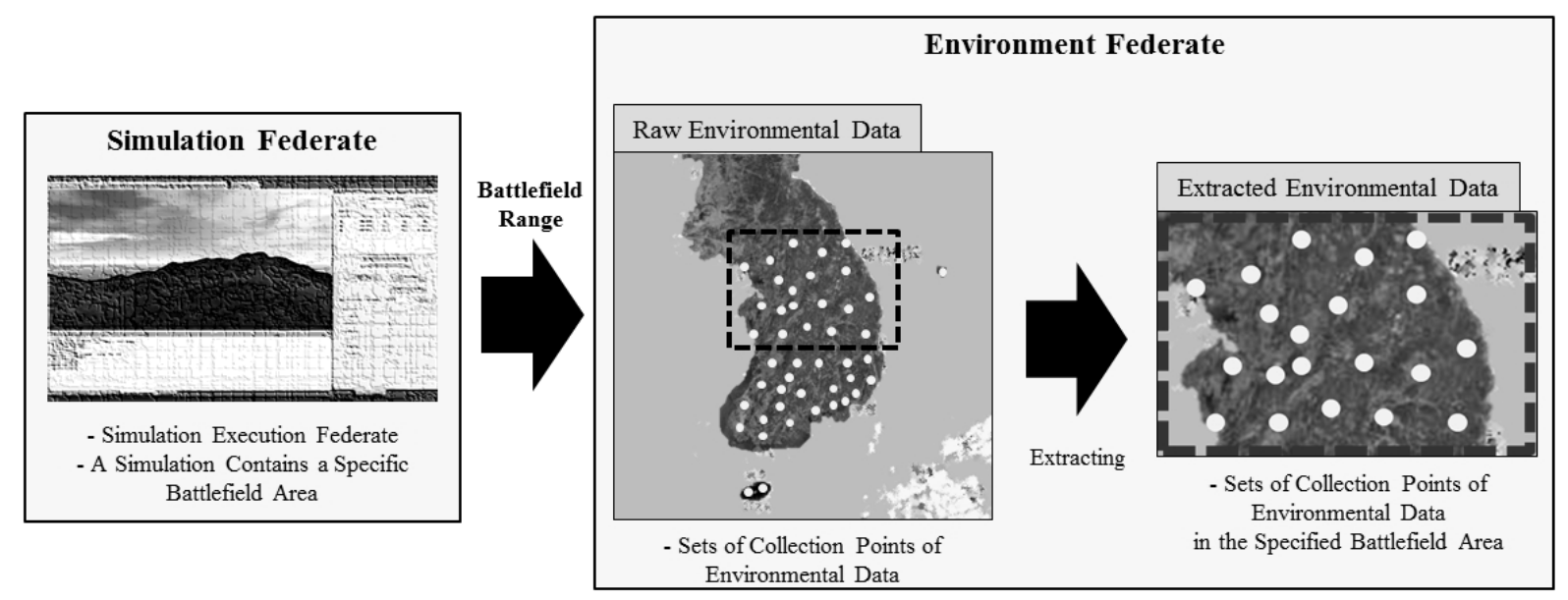

Figure 4: Searching the environmental data for the battlefield range.

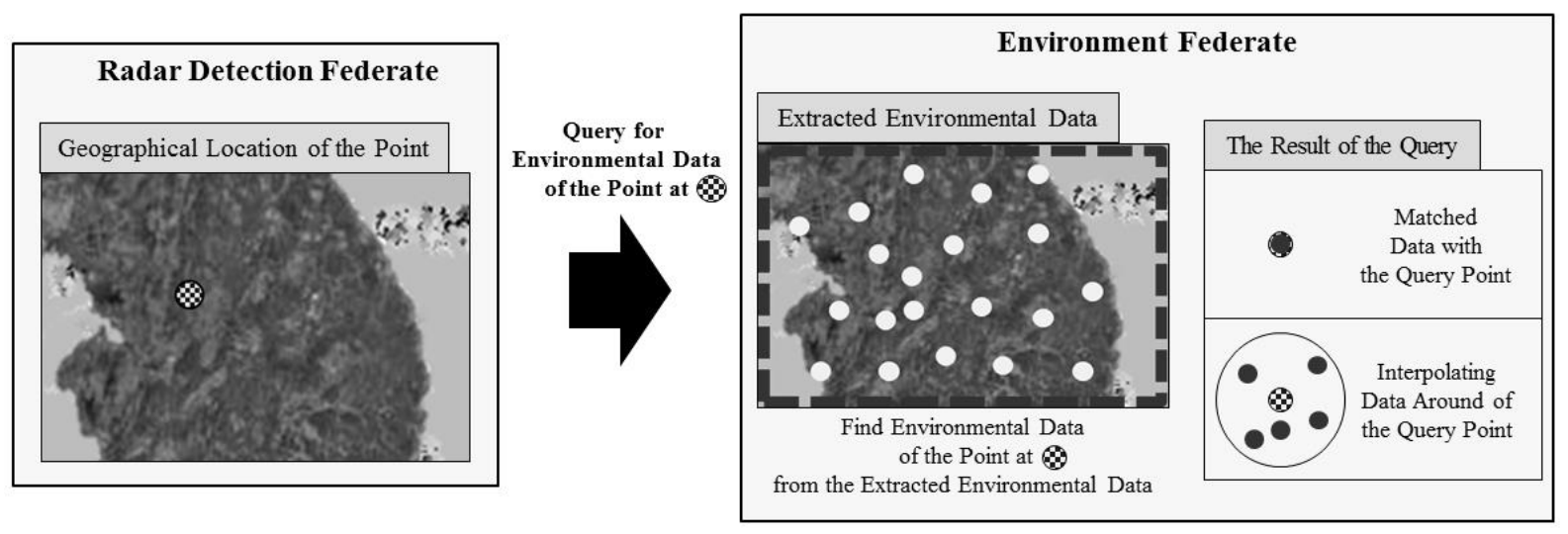

Figure 5: Searching the extracted data for the time and geographical location.

The extracted environmental data are used to search for the query from the radar detection federate. As depicted in Fig. 5, the radar detection federate publishes a query for the geographical location of the radar base. The environment federate subscribes to the query and finds environmental data for the query within the extracted data. If the environment federate finds a station that matches the point of the query, it publishes the data of that station as the result of the query. In many cases, however, it is not able to find a station that is equivalent to the point of the query. In that case, the environment federate derives the result via the interpolation of the data from stations that are around the query point, using methods that are preferred in meteorology, such as IDW (inverse distance weighting) or Kriging.

The empty GP table and radar specification published by the simulation federate are subscribed to by the radar detection federate. The empty GP table divides a detection range into distance ranges, each of which is indicated by a glimpse index. The radar specification contains information regarding the geographical location of the radar base and the frequency and transmission power of the radar. The radar detection federate publishes the geographical location of the radar base in order to acquire environmental data from the environment federate, and it then calculates the attenuation of the radio waves using that data. The radar detection federate computes the GP for every glimpse index of the empty GP table, and it applies the calculated results for the attenuation to the GPs in order to determine the GPs' reflection of environmental effects. The GP table, which includes the GPs' reflection of the environmental effects, is the complete GP table that is published for application to the anti-air engagement simulation. This entire process is presented graphically in Fig. 6. 


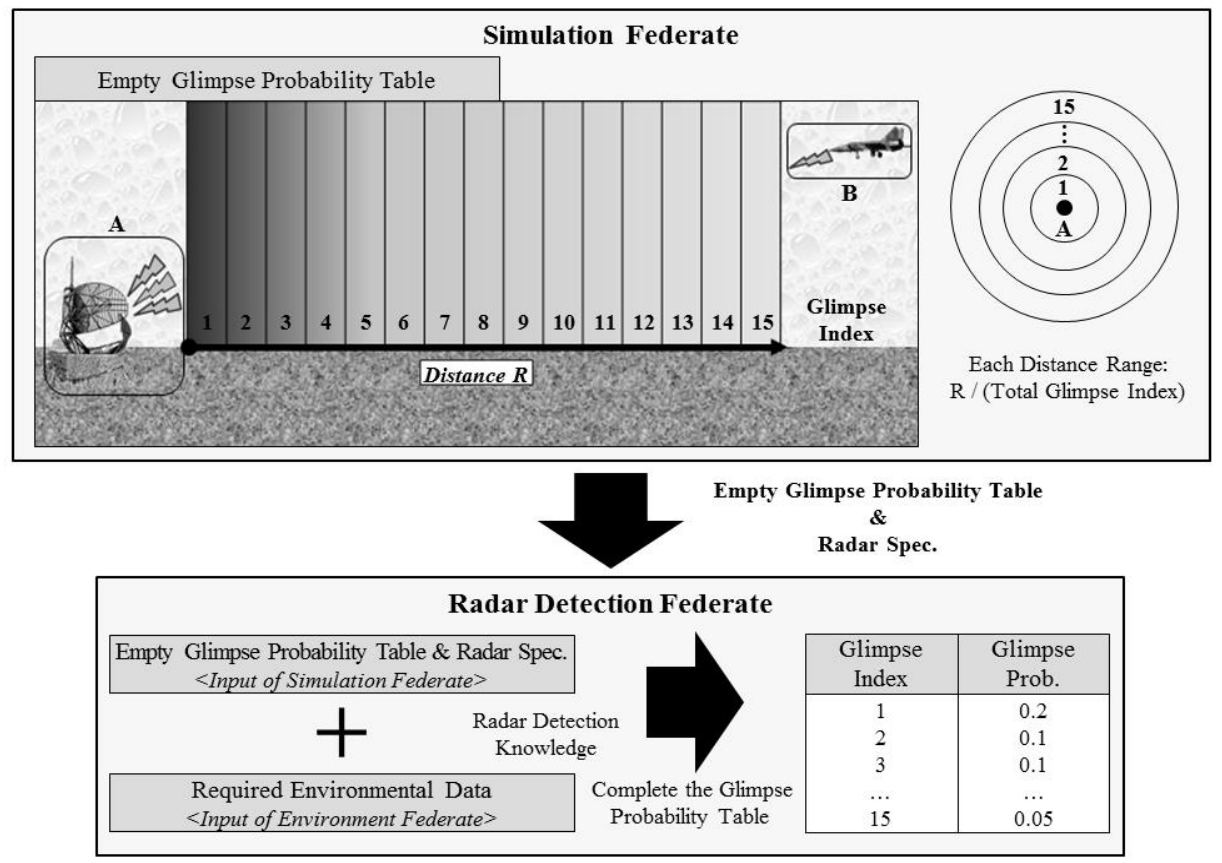

Figure 6: Construction process of GP table.

Finally, the simulation federate subscribes to the complete GP table and applies it to the aircraft detection probabilities of the radar base in the anti-air engagement simulation. The radar base checks the distance between the radar base and an aircraft, and identifies a GP at the equivalent distance from the GP table. The radar base is then able to determine the location of the aircraft based on the GP corresponding to that distance. Fig. 7 graphically describes the aircraft detection of a radar base as applied to the complete GP table in the simulation.

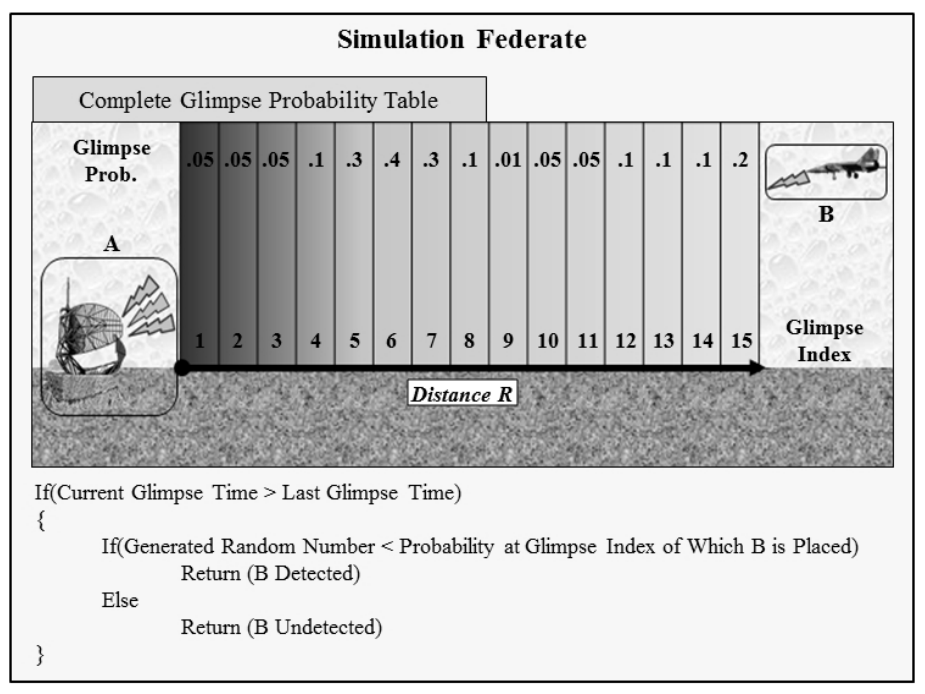

Figure 7: Application of GP table to the simulation

\section{DEMONSTRATION}

In order to demonstrate the proposed framework, this paper implements an example simulation system that is based on the framework. The federates, which are the components of the federation that are designed in the proposed framework, as discussed in the previous 
section, are implemented as applications that have an RTI connection interface. In the example system here, the federates complete the GP table by means of the data interchange defined in the framework, and the simulation federate executes engagement simulations using the complete GP table. All the federates were developed using the $\mathrm{C}++$ programming language and MFC (Microsoft Foundation Class) library. The simple functional specifications of the federates of the example system are as listed below.

\section{- Environment federate}

$\checkmark$ Contains atmospheric data of Korea's meteorological administration

$\checkmark \quad$ Extracts environmental data for a specified range from the container

$\checkmark$ Interpolates environmental data for the estimation of environmental values in geographical locations that have unidentified values

$\checkmark$ Publishes environmental values when it subscribes to a geographical location

\section{- Radar detection federate}

$\checkmark \quad$ Computes the degree of rain, vapour, and oxygen attenuation using environmental data

$\checkmark \quad$ Subscribes to the frequency and power of a radar base and an empty GP table

$\checkmark \quad$ Publishes the geographical location of a radar base and subscribes to the environmental data from the location of the radar.

$\checkmark$ Calculates the radar detection probabilities along the distance range (a demo equation is used in this study) and applies the three types of attenuation to the probabilities in order to complete the construction of the GP table

$\checkmark$ Publishes the complete GP table

\section{$\underline{\text { - Simulation federate }}$}

$\checkmark \quad$ Visualizes engagement simulations using OGRE3D (Object-oriented Graphics Rendering Engine $3 D$ ), which is a $C++$ based open visualization engine library

$\checkmark$ Configures the geographical location, frequency, and power of a radar base and battlefield range

$\checkmark$ Publishes the battlefield range

$\checkmark \quad$ Publishes the frequency and power of a radar device

$\checkmark$ Publishes the geographical location of a radar base, the unit distance range, and the maximum detection distance as an empty GP table

$\checkmark$ Subscribes to the complete GP table

$\checkmark$ Generates a random number and compares that number to the probability in the GP table (if the random number is larger than the GP, an aircraft has been detected)

The example system executes simulations for the example scenario (explained below) graphically presented in Fig. 8. Enemy aircraft are generated in the aircraft generation zone and are flying across an area that is under surveillance by an ally's surface ship, in order to reach its destination zone, which is on the opposite side of the generation zone.
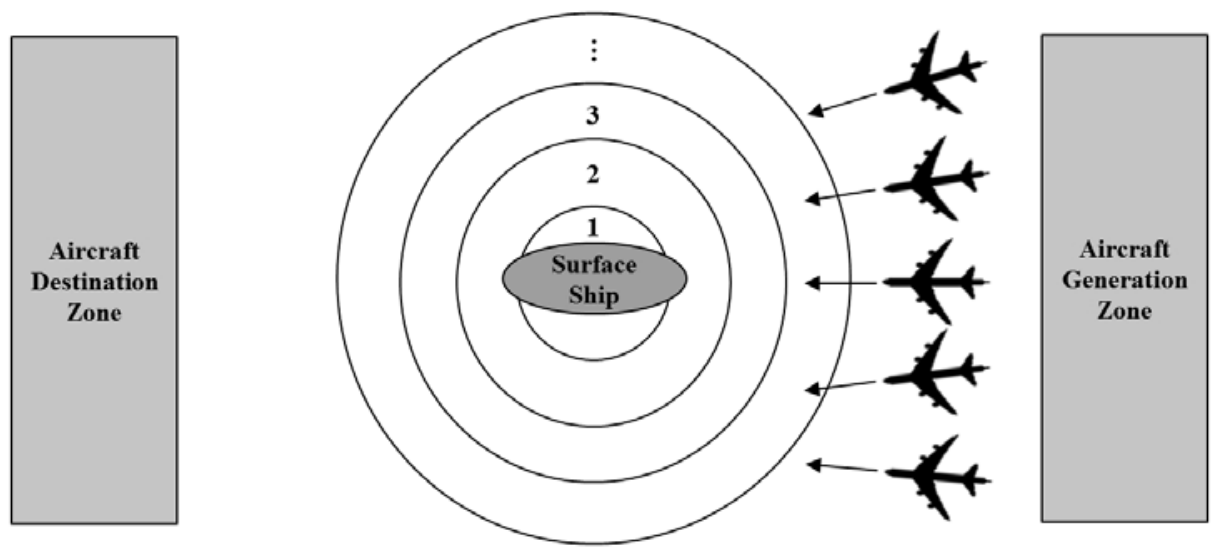

Figure 8: Example simulation scenario. 
If the surface ship detects enemies, it launches missiles at them, and the missiles track the enemies in order to shoot them down. The aircraft can either be destroyed by the missiles, or it can survive and arrive at its destination. For the simulation of the example scenario, the surface ship calculates the distances between itself and every aircraft, and derives the glimpse probabilities at each distance in the GP table. The surface ship launches missiles at enemies that are detected, based on the GP. After a missile is launched from a surface ship, it flies toward the targeted enemy aircraft. A missile has a maximum tracking distance and flight speed, and an aircraft also has a flight speed. If a missile travels its maximum tracking distance, it stops tracking the target and the targeted aircraft can arrive at its destination zone.
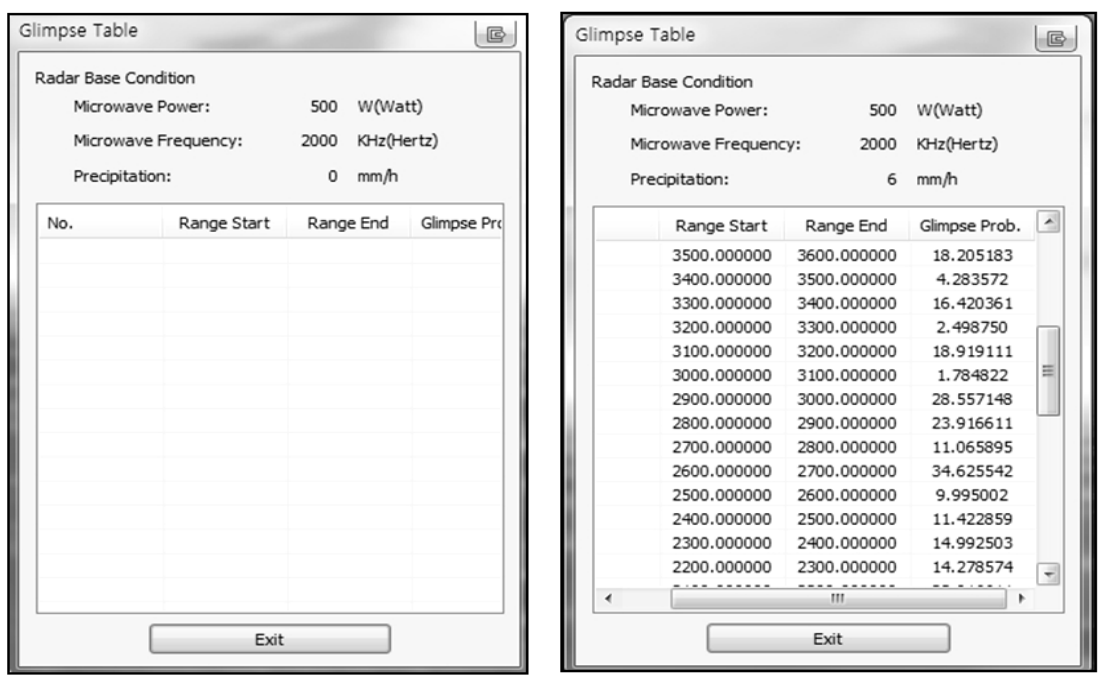

Figure 9: Construction of GP table; empty GP table (left), complete GP table (right).

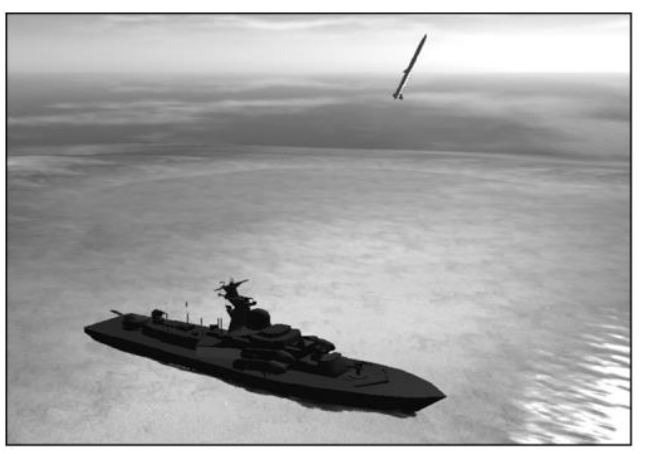

(a) The surface ship launching a missile

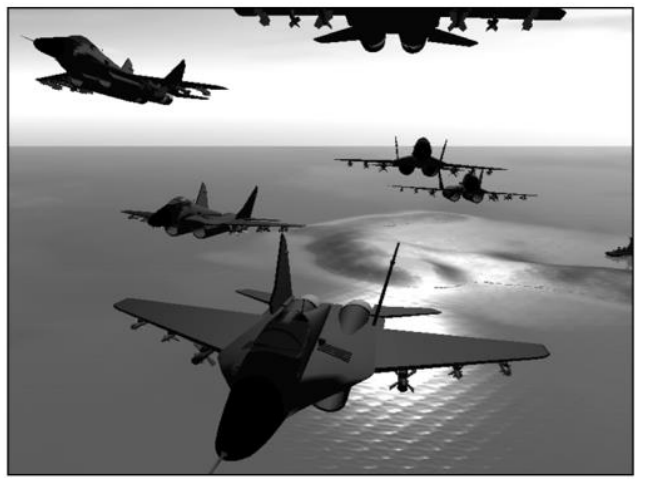

(c) Survived aircraft from missiles

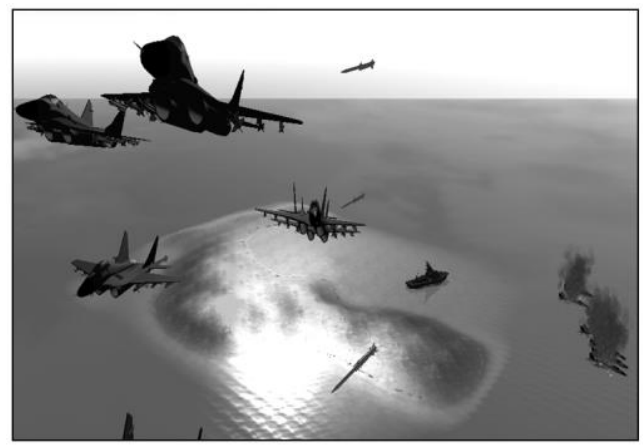

(b) Missiles tracing enemy aircraft

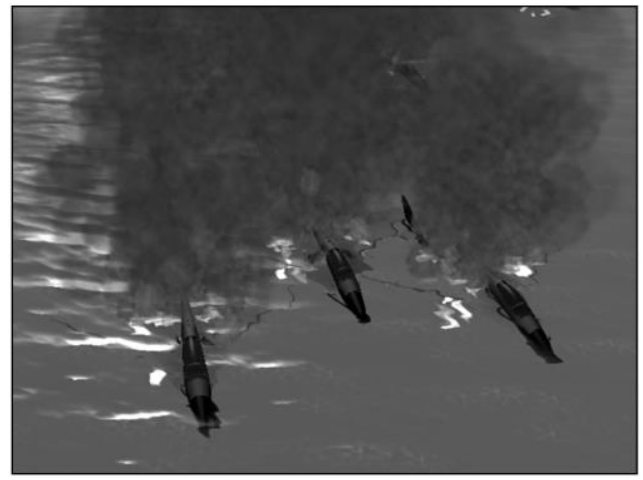

(d) Destroyed aircraft by missiles

Figure 10: 3D visualization of simulation. 
For the simulation of the example scenario, the simulation federate configures the initial scenario conditions, including the battlefield time; the enemy aircraft generation and destination zone; the total number of generated enemy aircraft; and the geographical location, power, and frequency of the radar base. The simulation federate publishes the empty GP table and battlefield information, and it subscribes to the complete GP table. The GP table construction is described in Fig. 9. When the simulation federate obtains the complete GP table, it is able to execute the anti-air engagement simulation. Fig. 10 is a representation of the anti-air engagement simulation obtained by 3D visualization, which shows key stages of the execution of the simulation, such as the launching of missiles and the tracking of target aircraft. The result stage of the simulation presented in Fig. 11 lists the enemy aircraft that survived, and those that were destroyed, and the missiles that exploded, and those that exhausted their tracking limit.

\begin{tabular}{|c|c|c|c|c|c|c|c|c|c|c|c|}
\hline \multicolumn{6}{|c|}{ Battle Entity List: } & \multicolumn{6}{|l|}{ Weapon Entity List: } \\
\hline Force Side & Entity ID & Entity Name & Position & State & A & Entity ID & Parent ID & Target ID & Position & State & A \\
\hline RED & OFFENSE_... & OFFENSE_... & $22556,774 \ldots$ & Idle & & RADAR_BASEMENT_RA... & RADAR_BA... & OFFENSE_... & No Where & Time Over & \\
\hline RED & OFFENSE_... & OFFENSE_... & $21741,830 \ldots$ & Idle & & RADAR_BASEMENT_RA... & RADAR_BA... & OFFENSE_... & No Where & Time Over & \\
\hline RED & OFFENSE_... & OFFENSE_... & $21067,959 \ldots$ & Idle & & RADAR_BASEMENT_RA... & RADAR_BA... & OFFENSE_... & No Where & Time Over & \\
\hline RED & OFFENSE_... & OFFENSE_... & $21263,916 \ldots$ & Idle & & RADAR_BASEMENT_RA... & RADAR_BA... & OFFENSE_... & No Where & Time Over & \\
\hline RED & OFFENSE_... & OFFENSE_... & $22744,943 \ldots$ & Idle & & RADAR_BASEMENT_RA... & RADAR_BA... & OFFENSE_... & No Where & Time Over & \\
\hline RED & OFFENSE_... & OFFENSE_... & $23718,763 \ldots$ & Idle & & RADAR_BASEMENT_RA... & RADAR_BA... & OFFENSE_... & No Where & Time Over & \\
\hline RED & OFFENSE_... & OFFENSE_... & $23305,854 \ldots$ & Idle & & RADAR_BASEMENT_RA... & RADAR_BA... & OFFENSE_... & No Where & Time Over & \\
\hline RED & OFFENSE_... & OFFENSE_... & $24908,9,6 \ldots$ & Destroyed & & RADAR_BASEMENT_RA... & RADAR_BA... & OFFENSE_... & No Where & Exploded & \\
\hline RED & OFFENSE_... & OFFENSE_... & $22099,17, \ldots$ & Destroyed & & RADAR_BASEMENT_RA... & RADAR_BA... & OFFENSE_... & No Where & Exploded & \\
\hline RED & OFFENSE_... & OFFENSE_... & $20769,9,2 \ldots$ & Destroyed & & RADAR_BASEMENT_RA... & RADAR_BA... & 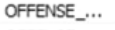 & No Where & Exploded & \\
\hline RED & OFFENSE_... & OFFENSE_... & $22743,18, \ldots$ & Destroyed & & RADAR_BASEMENT_RA... & RADAR_BA... & OFFENSE_... & No Where & Exploded & $\equiv$ \\
\hline RED & OFFENSE_... & OFFENSE_... & $22581,10, \ldots$ & Destroyed & & RADAR_BASEMENT_RA... & RADAR_BA... & OFFENSE_... & No Where & Exploded & \\
\hline RED & OFFENSE_... & OFFENSE_... & $24324,31, \ldots$ & Destroyed & & RADAR_BASEMENT_RA... & RADAR_BA... & OFFENSE_... & No Where & Exploded & \\
\hline RED & OFFENSE_... & OFFENSE_... & $24596,13, \ldots$ & Destroyed & & RADAR_BASEMENT_RA... & RADAR_BA... & OFFENSE_... & No Where & Exploded & \\
\hline RED & OFFENSE_... & OFFENSE_... & $22758,25, \ldots$ & Destroyed & & RADAR_BASEMENT_RA... & RADAR_BA... & OFFENSE_... & No Where & Exploded & \\
\hline i & & III & & 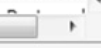 & & RADAR_BASEMENT_RA... & RADAR_BA... & OFFENSE_... & $\begin{array}{l}\text { No Where } \\
\text {...... }\end{array}$ & Exploded & - \\
\hline
\end{tabular}

Figure 11: Result state of simulation.

In this study, we performed experiments under different environmental and simulation conditions using the example system. As the results of the experiments show, the ratios of surviving aircraft to destroyed aircraft change with the attenuation of the radio waves and the specifications of a missile. In the experiments, the specifications of a radar base were constant values of a frequency of $20 \mathrm{GHz}$ and $2000 \mathrm{~kW}$ of power, and 50 enemy aircrafts were generated in the duration of each experiment. Table IV illustrates the average results obtained from ten executions under each experimental condition.

Table IV: Experiment results from simulations of the example system.

\begin{tabular}{|c|c|c|c|c|c|c|c|c|c|}
\hline \multirow{2}{*}{\begin{tabular}{|c|} 
Battlefield \\
$\begin{array}{c}\text { Time } \\
(\mathrm{yy} / \mathrm{mm} / \mathrm{dd} / \mathrm{hh}\end{array}$
\end{tabular}} & \multicolumn{2}{|c|}{ Radar Location } & \multicolumn{3}{|c|}{ Attenuation } & \multicolumn{2}{|c|}{ Missile } & \multicolumn{2}{|c|}{ Simulation Result } \\
\hline & $\begin{array}{c}\text { Latitude } \\
\left(\text { decimal }^{\circ}\right)\end{array}$ & $\begin{array}{c}\text { Longitude } \\
\left(\text { decimal }^{\circ}\right)\end{array}$ & $\begin{array}{l}\text { Rain } \\
(\mathrm{dB})\end{array}$ & $\begin{array}{c}\text { Vapour } \\
\text { (dB) }\end{array}$ & $\begin{array}{c}\text { Oxygen } \\
\text { (dB) }\end{array}$ & Range & Speed & Survived & Destroyed \\
\hline $91 / 06 / 13 / 09$ & 38.2 & 128.44 & 0.277 & 0.805 & 0.010 & Long & Fast & 24.7 & 25.3 \\
\hline $91 / 06 / 13 / 09$ & 38.2 & 128.44 & 0.277 & 0.805 & 0.010 & Short & Fast & 29.9 & 30.1 \\
\hline $91 / 06 / 13 / 09$ & 38.2 & 128.44 & 0.277 & 0.805 & 0.010 & Long & Slow & 35.1 & 14.9 \\
\hline $91 / 06 / 13 / 09$ & 38.2 & 128.44 & 0.277 & 0.805 & 0.010 & Short & Slow & 38.4 & 11.6 \\
\hline $94 / 09 / 10 / 21$ & 37.5 & 126.2 & 0.060 & 1.133 & 0.010 & Long & Fast & 26.1 & 23.9 \\
\hline $94 / 09 / 10 / 21$ & 37.5 & 126.2 & 0.060 & 1.133 & 0.010 & Short & Fast & 30.5 & 29.5 \\
\hline $94 / 09 / 10 / 21$ & 37.5 & 126.2 & 0.060 & 1.133 & 0.010 & Long & Slow & 36.2 & 13.8 \\
\hline $94 / 09 / 10 / 21$ & 37.5 & 126.2 & 0.060 & 1.133 & 0.010 & Short & Slow & 38.8 & 11.2 \\
\hline $00 / 08 / 13 / 18$ & & & $\overline{0.0}$ & 0.761 & 0.009 & Long & Fast & 18.2 & 31.8 \\
\hline $00 / 08 / 13 / 18$ & 37.1 & 130.1 & 0.0 & 0.761 & 0.009 & Short & Fast & 22.1 & 27.9 \\
\hline $00 / 08 / 13 / 18$ & 37.1 & 130.1 & 0.0 & 0.761 & 0.009 & Long & Slow & 31.6 & 18.4 \\
\hline $00 / 08 / 13 / 18$ & 37.1 & 130.1 & 0.0 & 0.761 & 0.009 & Short & Slow & 32.3 & 17.7 \\
\hline
\end{tabular}




\section{CONCLUSION}

Although the reflection of environmental effects in the DM\&S has been considered to be a key factor in future military simulations, its efficacy is hindered by the difficulty in applying a synthetic environment to an engagement simulation. The reason for this difficulty is that continuous systems, which commonly require the synthetic environment such as detection systems, are not proper for direct use in the discrete-event based combat simulation. The framework proposed in this paper was developed in order to bridge this gap between continuous detection systems and the discrete combat simulation system. The framework was applied to an anti-air engagement simulation that would reflect the environmental effects of the atmosphere in the radar detection system used in this study. In conclusion, the anti-air engagement simulation system based on the framework proposed in this paper performs simulations using the GP table for synthetic-environment-based radar detection stages, and the framework demonstrates its ability to bridge the problematic gap in the implementation of the example system.

One limitation of this study, though, is that the radar base location is constant during the simulation. Thus, the GP is created once, at the beginning of the simulation. In future work, it would be helpful if the location of the radar base is changeable, and the GP table is re-created, based on the movement of the radar base.

\section{ACKNOWLEDGEMENTS}

This work was supported by the Defense Acquisition Program Administration (DAPA) and the Agency for Defense Development (ADD) under the Contract No. UD110006MD (DAPA), UD100009DD \& UD120035JD (ADD). The authors wish to express sincere gratitude for the financial support.

\section{REFERENCES}

[1] Smith, R. D. (1998). Essential Techniques for Military Modeling \& Simulation, Proceedings of the 1998 Winter Simulation Conference, 805-812, doi:10.1109/WSC.1998.745067

[2] Herdman, R. C. (1995). Distributed Interactive Simulation of Combat, Office of Technology Assessment of U. S. Congress, Washington DC

[3] Keane, J. F.; Lutz, R. R.; Myers, S. E.; Coolahan, J. E. (2000). An architecture for simulation based acquisition, Johns Hopkins APL Technical Digest, Vol. 21, No. 3, 348-358

[4] Hawley, P. A.; Blauwkamp, R. A. (2010). Six-degree-of-freedom digital simulations for missile guidance, navigation, and control, Johns Hopkins APL Technical Digest, Vol. 29, No. 1, 71-84

[5] Republic of Korea Sergeant's Association (2007). History of War, Global Book, Korea

[6] Defense Modeling and Simulation Office (1995). Modeling and Simulation Master Plan, DoD 5000.59-P, USA

[7] Lwin, T.; Vu, N. A.; Lee, J. W.; Kim, S. H. (2012). A distribute web-based framework for helicopter rotor blade design, Advances in Engineering Software, Vol. 53, 14-22, doi:10.1016/j.advengsoft.2012.07.003

[8] Lee, C. G.; Park, S. C. (2014). Survey on the virtual commissioning of manufacturing systems, Journal of Computational Design and Engineering, Vol. 1, No. 3, 213-222, doi:10.7315/ JCDE.2014.021

[9] Kaban, A. K.; Othman, Z.; Rohmah, D. S. (2012). Comparison of dispatching rules in job-shop scheduling problem using simulation: a case study, International Journal of Simulation Modelling, Vol. 11, No. 3, 129-140, doi:10.2507/IJSIMM11(3)2.201

[10] Reddy, B. S. P.; Rao, C. S. P. (2011). Flexible manufacturing systems modelling and performance evaluation using AutoMod, International Journal of Simulation Modelling, Vol. 10, No. 2, 78-90, doi:10.2507/IJSIMM10(2)3.176 
[11] Gwenda, F. (2006). Adapting COTS games for military experimentation, Simulation \& Gaming, Vol. 37, No. 4, 452-465, doi:10.1177/1046878106291670

[12] Park, S. C.; Kwon. Y.; Seong, K.; Pyun, J. J. (2010). Simulation framework for small scale engagement, Computers \& Industrial Engineering, Vol. 59, No. 3, 463-472, doi:10.1016/j.cie.2010.06.003

[13] Yildirim, U. Z.; Tansel, B. C.; Sabuncuoglu, I. (2009). A multi-modal discrete-event simulation model for military deployment, Simulation Modelling Practice and Theory, Vol. 17, No. 4, 597611, doi:10.1016/j.simpat.2008.09.016

[14] Son, M.-J.; Kim, T.-W. (2012). Torpedo evasion simulation of underwater vehicle using fuzzylogic-based tactical decision making in script tactics manager, Expert Systems with Applications, Vol. 39, No. 9, 7995-8012, doi:10.1016/j.eswa.2012.01.113

[15] Wilcox, P. A.; Burger, A. G.; Hoare, P. (2000). Advanced distributed simulation: a review of developments and their implication of data collection and analysis, Simulation Modelling Practice and Theory, Vol. 8, No. 3-4, 200-231, doi:10.1016/S0928-4869(00)00023-9

[16] ES310 (1998). Introduction to naval weapon engineering, Military Analysis Network of Federation of American Scientists, Radar Fundamentals Section

[17] Wikipedia. Radio Propagation, from http://en.wikipedia.org/wiki/Radio_propagation, accessed on 01-06-2013

[18] Paris, D. T.; Hurd, F. K. (1969). Basic Electromagnetic Theory, McGraw Hill, New York

[19] IEEE Std 1516TM (2010). IEEE Standard for Modeling and Simulation (M\&S): High Level Architecture (HLA) - Framework and rules, IEEE Computer Society, New York

[20] M\&SCO. Description of M\&SCO, from http://www.msco.mil/descMSCO.html, accessed on 0106-2013

[21] Australian Defense Simulation Office (2004). Distributed simulation guide, Department of Defence, Canberra

[22] Coulouris, G.; Dollimore, J.; Kindberg, T.; Blair, G. (2012). Distributed Systems: Concepts and Design (Fifth ed.), Addison-Wesley, Boston

[23] Defense Modeling and Simulation Office (2003). RTI 1.3-Next generation programmer's guide version 6.4.3, Department of Defense, USA

[24] IEEE Std 1516TM (2010). IEEE Standard for Modeling and Simulation (M\&S): High Level Architecture (HLA) - Federate Interface Specification, IEEE Computer Society, New York

[25] ITU-R (2005). ITU-R P. 838-3: Specific Attenuation Model for Rain for Use in Prediction Methods, Radiocommunication sector of ITU

[26] ITU-R (2012). ITU-R P. 676-9: Attenuation by Atmospheric Gases, Radiocommunication sector of ITU

[27] Lee, I. K.; Park, D. C.; Yoo, T. S.; Myung, R. H.; Lim, J. S.; Cho, Y. H.; Kang, H. C. (2006). Radar Engineering and Applications in Electronic Warfare, Daeyoung, Seoul

[28] Driels, M. (2004). Weaponeering: Conventional Weapon System Effectiveness (first ed.), American Institute of Aeronautics and Astronautics, Reston

[29] Korea Military Academy (2003). Weapons System Engineering, Bookshill, Korea 